\title{
Bemerkung zur Theorie der zahlentheoretischen Ableitungen.
}

Von R. Daublebsky von Sterneck in Wien.

Besteht zwischen den Functionen $\Theta$ und $\psi$ die Relation:

$$
\sum_{n} \theta(d)=\psi(n)
$$

wobei sich das Summenzeichen ïber alle Theiler der ganzen Zahl $n$ erstreckt, so bezeichnet Herr Bugajeff $\psi(n)$ als das zahlentheoretische Integral der Function $\theta(n) . \theta(n)$ ist dann die zahlentheoretische Ableitung der Function $\psi(n)$, wofür das Symbol gebraucht wird:

$$
\theta(n)=D \biguplus(n)
$$

Es handelt sich nun darum, bei gegebener Function $\psi(n)$ die Function $\theta(n)$ zu bestimmen.

Herr Bugajeff führt den Beweis des hierauf bezüglichen Theoremes successive, indem $n$ zuerst als Primzahl, dann als Potenz einer Primzahl, endlich als beliebig zusammengesetzte Zahl angenommen wird. Ich versuche nun, für eine beliebig zusammengesetzte Zahl $n$ den Beweis direct zu führen.

Bezeichnet $\left[\begin{array}{l}n \\ k\end{array}\right]$ die größte, in $\frac{n}{k}$ enthaltene ganze Zahl, so erhalten wir durch Summierung der Gleichung:

$$
\sum_{n} \theta(d)=\psi(n)
$$

über die Reihe der natürlichen Zahlen von 1 bis $n$ den Ausdruck:

$$
\psi(1)+\psi(2)+\ldots+\psi(n)=\theta(1)\left[\frac{n}{1}\right]+\theta(2)\left[\frac{n}{2}\right]+\ldots+\theta(n)\left[\begin{array}{l}
n \\
\frac{n}{n}
\end{array}\right] .
$$

Denn $\left[\begin{array}{l}n \\ \vec{k}\end{array}\right]$ bezeichnet ja zugleich, wieviele Zahlen der Reihe $1,2, \ldots n$ durch $k$ theilbar sind. 
Setzen wir zur Abkürzung:

$$
\psi(1)+\psi(2)+\ldots+\psi(n)=F(n)
$$

so liefert die eben gewonnene Gleichung für die Werte: $n=1,2, \ldots n$ das System von $n$ linearen Gleichungen:

$$
\begin{aligned}
& F(1)=\theta(1)\left[\frac{1}{1}\right] \\
& F(2)=\theta(1)\left[\frac{2}{1}\right]+\theta(2)\left[\frac{2}{2}\right] \\
& F(3)=\theta(1)\left[\frac{3}{1}\right]+\theta(2)\left[\begin{array}{l}
3 \\
\frac{2}{2}
\end{array}\right]+\theta(3)\left[\frac{3}{3}\right] \\
& \cdot \cdot \cdot \cdot \cdot \cdot \cdot \cdot \cdot \cdot \cdot \cdot \\
& F(n)=\theta(1)\left[\frac{n}{1}\right]+\theta(2)\left[\frac{n}{2}\right]+\ldots+\theta(n)\left[\frac{n}{n}\right]
\end{aligned}
$$

Bestimmen wir daraus die Unbekannte $\theta(n)$, so erhalten wir, da die Determinante des Nenners sich auf die positive Einheit reducirt:

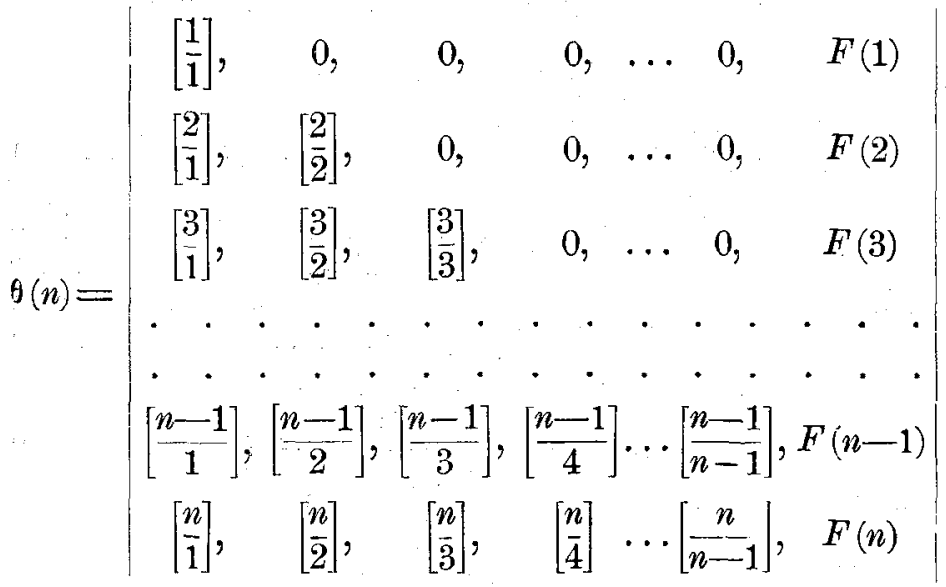

Durch Subtraction der ersten Zeile von der zweiten, der zweiten von der dritten, u. s. f., endlich der $n-1^{\text {ten }}$ von der $n^{\text {ten }}$, nimmt die $\lambda^{\text {to }}$ Colonne dieser Determinante, da $\left[\frac{k}{\mu}\right]-\left[\frac{k-1}{\mu}\right]$ den Wert 1 oder 0 hat, je nachdem $k$ durch $\mu$ theilbar ist oder nicht, folgende Gestalt an: Zuerst stehen in derselben $\lambda-1$ Glieder mit dem Werte 0 , dann 1 Glied mit dem Werte 1 ; dann wieder $\lambda-1$ Glieder mit dem Werte 0 und 1 Glied mit dem Werte 1, u. s. f. In der letzten Colonne stehen die Werte $\psi(1), \psi(2), \ldots \psi(n)$. 
Die Determinante sieht daher folgendermaßen aus:

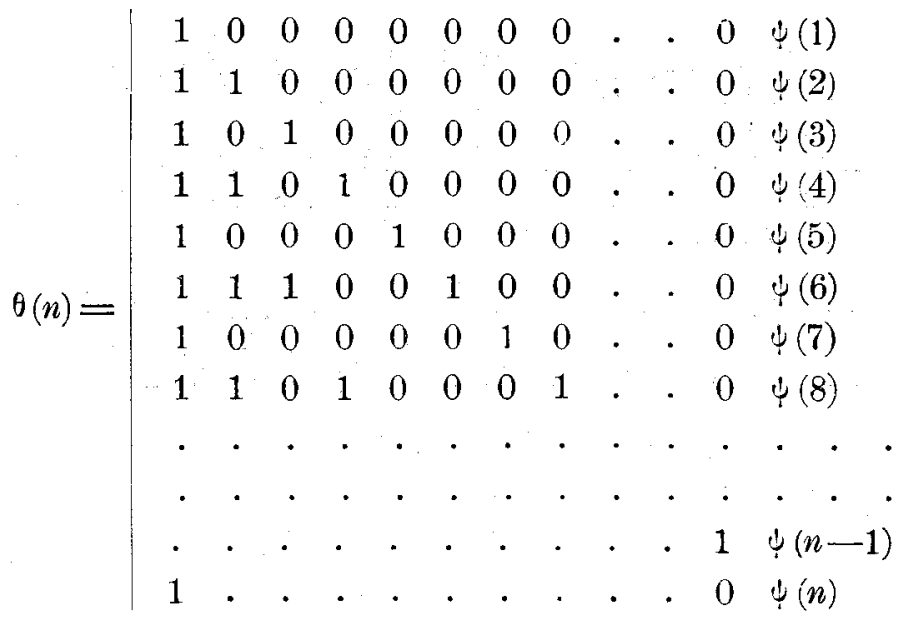

Diese Determinante lässt sich, wie nun gezeigt werden soll, leicht auswerten.

Betrachten wir irgend eine Horizontalzeile derselben, etwa die $k^{\text {te }}$. In derselben steht an allen jenen Stellen der Wert 1, deren Stellenzeiger Theiler von $k$ sind, an allen übrigen Stellen der Wert 0; das letzte Glied der Zeile ist $U(k)$.

Nehmen wir an, $n$ habe, in Primfactoren zerlegt, die Gestalt:

$$
n=a_{1}^{\alpha_{1}} a_{2}^{a_{2}} \ldots a_{\nu}^{\alpha_{\nu}}
$$

und wird mit $d^{(\varrho)}$ eine Zahl bezeichnet, welche aus $n$ hervorgeht, wenn man darin $\rho$ Exponenten $\alpha \mathrm{um}$ eine Einheit erniedrigt, die übrigen $\nu$ - $\rho$ Exponenten $\alpha$ ungeändert lässt, so ist die Anzahl der mit $d^{(\varrho)}$ bezeichneten Zahlen $\left(\begin{array}{l}v \\ p\end{array}\right)$.

Subtrahieren wir nun in der Determinante von der letzten Zeile die sämmtlichen $d^{(1) \text { ten }}$ Zeilen, addieren die sämmtlichen $d^{(2) \text { ten }}$ Zeilen, subtrahieren die sämmtlichen $d^{(3) \text { ten }}$ Zeilen, u. s. f.

In der letzten Zeile steht an allen jenen Stellen, deren Stellenzeiger Theiler von $n$ sind, der Wert 1 ; ein Theiler von $n$ hat die Gestalt $a_{1}^{\varepsilon_{1}} a_{2}^{\varepsilon_{2}} \ldots a_{v^{v}}$, und es seien etwa $\mu$ der Größen $\varepsilon$ gleich, die übrigen $\nu-\mu$ kleiner als die entsprechenden $\alpha$; die Anzahl der Größen $d^{(\varrho)}$, welche durch diesen ins Auge gefassten Theiler von $n$ theilbar sind, ist dann offenbar $\left(\begin{array}{c}\nu-\mu \\ \rho\end{array}\right)$. 
Es tritt somit nach Ausführung unseres Processes an die dem betreffenden Theiler entsprechende Stelle der letzten Zeile:

$1-\left(\begin{array}{c}\nu-\mu \\ 1\end{array}\right)+\left(\begin{array}{c}\nu-\mu \\ 2\end{array}\right)-\left(\begin{array}{c}\nu-\mu \\ 3\end{array}\right)+-\ldots+(-1)^{\nu-\mu-1}\left(\begin{array}{c}\nu-\mu \\ \nu-\mu-1\end{array}\right)+(-1)^{\nu-\mu}\left(\begin{array}{c}\nu-\mu \\ \nu-\mu\end{array}\right)=0$.

Die Determinante reduciert sich daher auf das letzte Glied der letzten Zeile, multipliciert mit dessen Subdeterminante, die den Wert +1 hat. Wir erhalten somit:

$\theta(n)=\psi(n)-\Sigma \psi\left(d^{(1)}\right)+\Sigma \psi\left(d^{(2)}\right)-\Sigma \psi\left(d^{(3)}\right)+\ldots+(-1)^{\prime} d^{(v)}$,

wotür Herr Bugajeff die symbolische Bezeichnung verwendet:

$$
\theta(n)=\psi[?(n)]
$$

